\section{Optical scatter changes at the onset of apoptosis are spatially associated with mitochondria}

\author{
Robert M. Pasternack, Jing-Yi Zheng, and \\ Nada N. Boustany \\ Rutgers University, Department of Biomedical Engineering, \\ Piscataway, New Jersey 08854
}

\begin{abstract}
We combine optical scatter imaging (OSI) with fluorescence imaging of mitochondria to investigate the spatial relationship between the optical scatter signal and the location and structure of mitochondria within endothelial cells undergoing apoptosis. The OSI data corroborate our previous results showing a decrease in the intensity ratio of wide-to-narrow angle scatter [optical scatter image ratio (OSIR) ] during the first 60 min of apoptosis. In addition, we find here that this is followed by an increase in OSIR concurrent with mitochondrial fragmentation. We demonstrate that the dynamic change in light scattering is spatially associated with subcellular regions containing fluorescently labeled mitochondria, and remains absent from adjacent nonfluorescent regions dominated by other organelles. These results lend strong support to the hypothesis that mitochondria act as the source of the optical scatter changes measured at the onset of apoptosis. @ 2010 Society of Photo-Optical Instrumentation Engineers. [DOI: 10.1117/1.3467501]
\end{abstract}

Keywords: scattering; cells; biology; fluorescence; microscopy.

Paper 10255LR received May 11, 2010; revised manuscript received Jun. 25, 2010; accepted for publication Jun. 28, 2010; published online Aug. 23, 2010.

Programmed cell death (apoptosis) is regulated by a balance between pro-survival and pro-death factors that converge on the mitochondria. ${ }^{1}$ Mitochondrial permeabilization by proapoptotic proteins Bax and Bak is a critical event in the apoptosis pathway, as it allows cytochrome c release and downstream caspase activation, and cell death. ${ }^{1}$ In addition, Bax/Bax localization to the outer mitochondrial membrane occurs nearly simultaneously with Drp1-mediated mitochondrial fragmentation. ${ }^{2}$ From an optics standpoint, mitochondria are highly scattering and account for most of the light scattered by organelles in the cytoplasm. ${ }^{3}$ Thus, several studies have shown alterations in light scattering concurrent with alteration in mitochondrial function and structure during apoptosis. ${ }^{4-6}$ In this work, we extend these previous studies by demonstrating that optical scatter changes occurring within the first three hours of apoptosis are spatially registered with mitochondria, therefore lending significant support to the hypothesis that mitochondria act as the source of optical scatter changes early during apoptosis.

We used bovine aortic endothelial cells (BAEC, Clonetics Lonza, Chicago, Illinois) cultured on glass coverslips as pre-

Address all correspondence to: Nada Boustany. Tel: 732-445-4500 x6320; Fax: 732-445-3793; E-mail: nboustan@rci.rutgers.edu viously described. ${ }^{7}$ For fluorescence imaging, the cells were labeled with $100 \mathrm{nM}$ Mitotracker Green (Invitrogen, Carlsbad, California), which specifically localizes in the mitochondria. The coverslips were mounted and viewed in non- $\mathrm{CO}_{2}$-dependent Leibovitz L-15 medium (Invitrogen) supplemented with $10 \%$ fetal bovine serum (FBS) at room temperature and room air. Apoptosis was induced by exchanging the cells' normal viewing medium for viewing medium containing $1 \mu \mathrm{M}$ staurosporine (STS) (Sigma Chemical, Saint Louis, Missouri) prepared from a $4 \mathrm{mM}$ staurosporine stock solution in dimethyl sulfoxide (DMSO) (Sigma). Control studies consisted of loading L-15 $+10 \%$ FBS with only DMSO in the same volume in place of the STS solution. The apoptotic fate of BAEC treated with STS was separately confirmed by positive immunofluorescence of cleaved caspase 3 .

The cells were studied on the optical Fourier processing microscope described in Ref. 8, fitted and aligned with a $63 \times$ oil immersion objective with a numerical aperture (NA) of 1.4. Optically filtered images were acquired on a Cascade 512B charge-coupled device (CCD) (Roper Scientific, Trenton, New Jersey). Fourier filtering was achieved with a TI0.7XGADMD 1100 digital mirror device (DMD) (Texas Instruments, Dallas, Texas). We applied the variable-diameter irises to the DMD to implement our previous OSI method, ${ }^{9}$ which consists of taking the ratio of two central dark ground images collected at high and low NA. In the present setup, the high NA iris had an outer radius of 180 mirrors (corresponding to the objective's full NA=1.4). The low NA iris had an outer radius of 34 mirrors (corresponding to $\mathrm{NA}_{\text {low }}=0.26$ ). The beam block at the center of the irises was 16 mirrors wide and blocked an NA of 0.06. Images of background consisting of a glass coverslip with no sample were collected with the same high and low NA apertures. The dark-field images were processed as described previously ${ }^{9}$ by taking the ratio of the background-subtracted high and low NA images to generate ratiometric images encoding the OSIR, giving a measure of wide-to narrow angle scatter at each pixel. As demonstrated in Ref. 9, a larger OSIR is associated with smaller particle size. Based on our work with different cell types, we have found that the OSIR signal from mitotracker labeled cells is not significantly different compared with unlabeled cells.

OSI was applied in time intervals of 20 min over a threehour time after STS addition at $\mathrm{T}=0$. For cells treated only with DMSO (controls), we repeated the optical acquisition taking data every 50 to $60 \mathrm{~min}$. At each time point studied with the OSI technique, fluorescent images of the mitotrackerlabeled cells were acquired with a fluorescein isothiocyanate (FITC) filter cube (filter number 10, Carl Zeiss, Gottingen, Germany), and without Fourier spatial filtering, bypassing the DMD by sending the light through the trinocular port of the microscope to a CoolSnap CCD (Roper Scientific). Differential interference images of the cells were acquired on both cameras. This allowed for registration of the backgroundsubtracted dark-field images, and therefore the OSI images, with the DIC and fluorescence images. The cells were segmented manually and data analysis was confined to these cell segments.

Morphometric images encoding the OSIR are shown in Fig. 1 for a representative cell, along with DIC and fluores-

1083-3668/2010/15(4)/040504/3/\$25.00 @ 2010 SPIE 


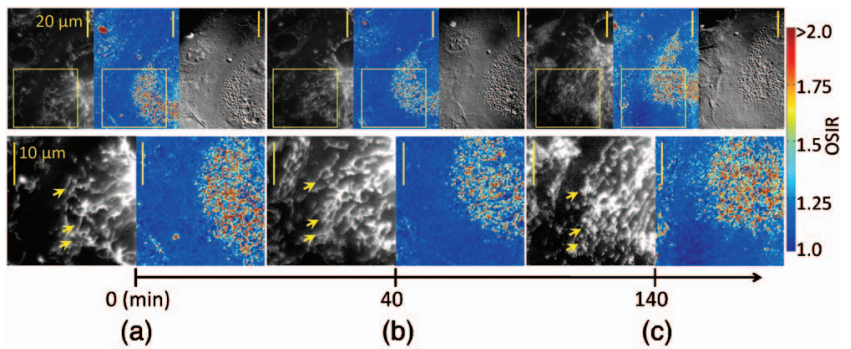

(a)

(b)

(c)

Fig. 1 Top row: fluorescence (left), OSI (middle), and differential interference contrast (right) images of a representative cell immediately after $1-\mu \mathrm{M}$ STS treatment at (a) $\mathrm{T}=0$, (b) $\mathrm{T}=40 \mathrm{~min}$, and (c) $\mathrm{T}=140 \mathrm{~min}$ after STS addition. Bottom row: magnified fluorescence and OSI insets showing mitochondrial fragmentation (arrows).

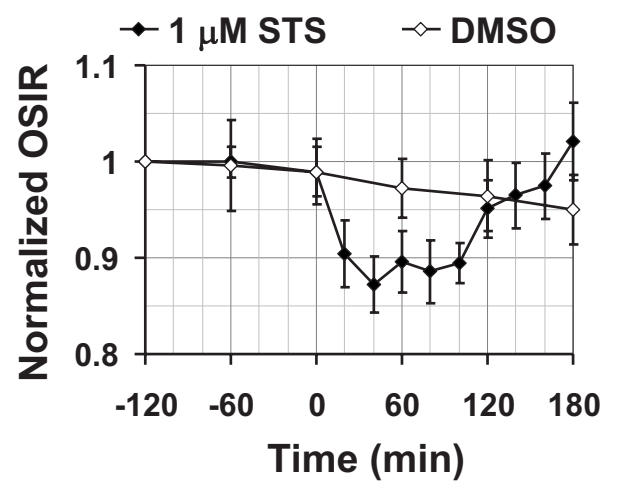

Fig. 2 OSIR change as a function of time for STS (black diamonds) and DMSO (white diamonds) treated cells. The value at each time point is the mean OSIR per cell normalized to the mean OSIR per cell at $\mathrm{T}=-120 \mathrm{~min}$. Error bars indicate \pm the $95 \%$ confidence interval of the mean. STS $(1 \mu \mathrm{M})$ or DMSO was added at $\mathrm{T}=0$. cence images at $\mathrm{T}=0$ (immediately after STS treatment), and 40 and $140 \mathrm{~min}$ after STS addition. For cells treated with STS, the average value of the OSIR per cell drops within the first $60 \mathrm{~min}$ of exposure to STS, and then steadily rises between 60 and 180 min (Fig. 2 black diamonds). In contrast, the OSIR remains largely unchanged for cells treated with only DMSO (Fig. 2 white diamonds). In the fluorescent images, mitochondria evolve from a long, filamentous morphology to shorter, smaller, and rounder shapes between 60 and $140 \mathrm{~min}$ [Fig. 1(c)]. This is consistent with previous observations of mitochondrial fragmentation in cells undergoing apoptosis. ${ }^{2}$ However, no gross changes in mitochondrial morphology could be discerned during the first $60 \mathrm{~min}$ of STS treatment.

Since the OSI method allows for spatially localizing the optical scatter changes within the cells, we investigated if the OSIR changes measured at the whole cell level (Fig. 2) could be specifically associated with subcellular regions containing mitochondria based on the fluorescence images of the labeled mitochondria. To correct for fluorescence background, each raw fluorescent image [Fig. 3(a), top panel] was filtered using a digital Gabor filterbank ${ }^{10}$ composed of filters with period $S$, equivalent to $0.9 \mu \mathrm{m}$ in the object, Gaussian envelope $\sigma_{s}=S / 4=0.225 \mu \mathrm{m}$, and orientations from 0 to $160 \mathrm{deg}$ with 20 -deg increments. Because the Gabor filter period $S$ is most sensitive to object features $\sim S / 2$ in size, $S$ was chosen to be twice the average short-axis diameter of mitochondria $(0.3$ to $0.6 \mu \mathrm{m}) .{ }^{11}$ The Gabor filtered fluorescent images were then summed to yield a background-corrected fluorescent image [Fig. 3(a), bottom panel].

At each time point, each cell segment was divided into two regions, one dominated by bright fluorescent mitochondria and the other by the remaining dim background consisting of

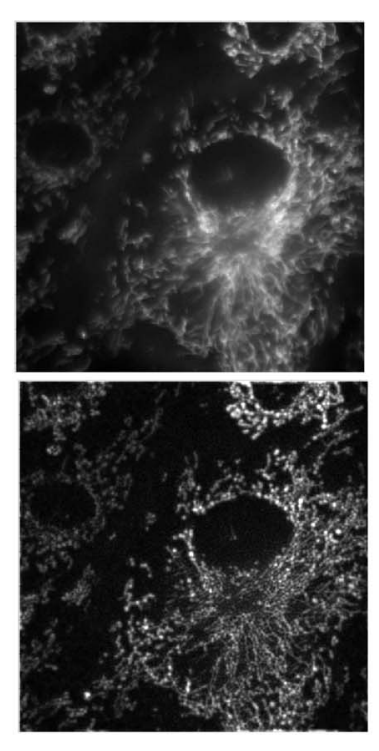

(a)

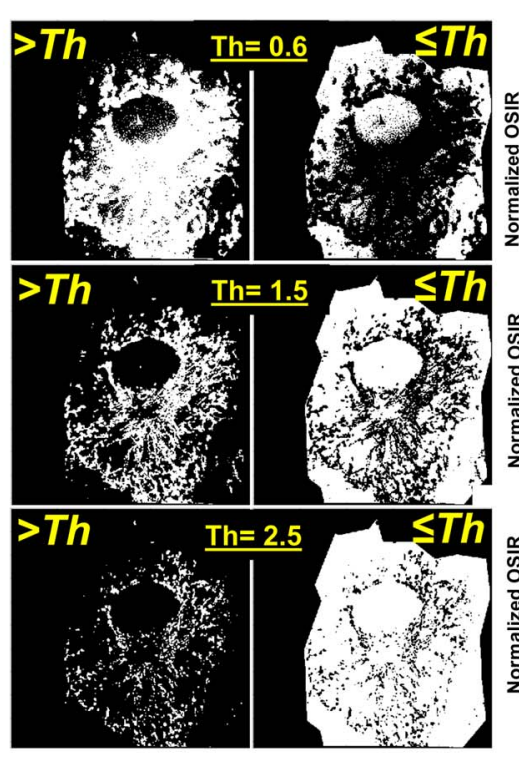

(b)

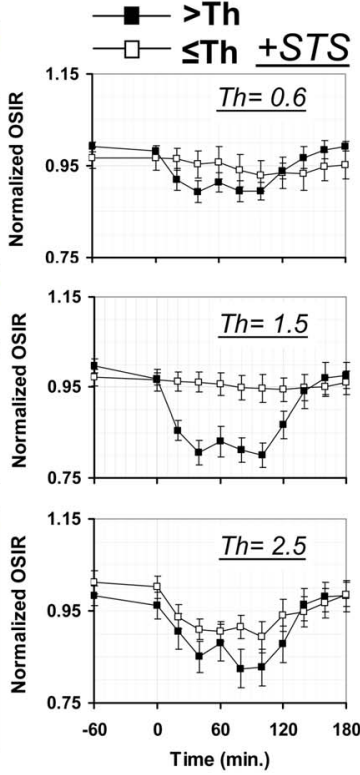

(c)
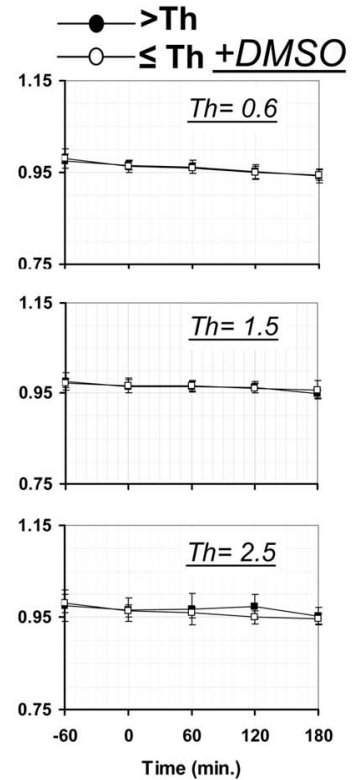

(d)

Fig. 3 (a) Representative raw fluorescence image (top panel) and Gabor filtered fluorescence image (bottom panel) used for thresholding study (images are shown for $\mathrm{T}=-60$ ). (b) Representative binary subcellular masks (shown for one cell) with fluorescence intensity above ( $>$ Th) or below ( $\leqslant$ Th) threshold. The threshold value (Th) was normalized to the average fluorescence intensity within a given cell segment. (c) and (d) Mean OSIR per masked cell $\pm 95 \%$ confidence interval as a function of time in the subcellular regions with fluorescence above threshold (filled squares) or below threshold (open squares). Data are shown for (c) STS and (d) DMSO treated cells, and for different values of Th. 
other unlabeled organelles [Fig. 3(b)] by applying an intensity threshold. This threshold was normalized to the average fluorescence intensity within a given cell segment. For example, if a threshold of $1(\mathrm{Th}=1)$ is chosen, all the fluorescent pixels with intensity values greater than the average intensity value for that cell are taken as fluorescent, and all the pixels with values less than or equal to the average intensity are taken as nonfluorescent. Once chosen, the normalized threshold value was the same for all the cell segments studied, and all pixels within each single cell segment were included in this analysis. The bright and dim fluorescent areas were then separately applied as a mask to the OSI images to compare the optical scatter changes within the brightly fluorescent areas rich in mitochondria, versus dim regions dominated by nonfluorescent organelles. Figure 3 shows the results of this analysis for three different choices of threshold values.

By choosing the threshold around $\mathrm{Th}=1.0$ to 1.5 , we were able to clearly separate the OSIR dynamic between the fluorescent areas above and below threshold for the STS treated cells [Fig. 3(c)]. The DMSO control studies [Fig. 3(d)] showed no significant changes in OSIR for all threshold values. These results strongly suggest that the optical scatter change is spatially localized in subcellular regions dominated by fluorescent mitochondria, and that regions with fluorescence intensity below $\mathrm{Th}=1.5$ and dominated by organelles other than mitochondria ("background") do not contribute appreciably to the measured scattering change. This value of $\mathrm{Th}=1.5$, which could separate well mitochondria from background in our system, could vary depending on the extent of fluorescence staining and the concentration of mitochondria within the cells under study. While the thresholding approach is computationally very simple, a more robust criterion could be found by utilizing a non-threshold-based segmentation algorithm (e.g. texture-based) to isolate the mitochondria-rich regions.

The increase in the OSIR signal between 60 and $120 \mathrm{~min}$ after STS addition was visibly accompanied by mitochondrial fragmentation, as observed in the fluorescence images. Thus, the increase in OSIR after 60 min likely reflects an overall decrease in particle size due to this fragmentation process. Current results ${ }^{12}$ utilizing orientation-dependent light scattering measurements support this hypothesis by showing a decrease in the degree of particle orientation at the time of mitochondrial fragmentation. The decrease in OSIR within the first hour of apoptosis reproduces our previous data, ${ }^{4,9}$ but is more difficult to interpret from a biophysical standpoint. While we show here that this decrease spatially registers with mitochondria, it was not accompanied by discernable changes in the fluorescent images. These scattering changes could reflect ultrastructural changes in the mitochondrial matrix. This is possible because the OSI technique, which does not rely on the visualization of the scatterers, can be sensitive to changes in particle size that are below the imaging resolution limit. ${ }^{9}$ In addition, in our previous work, we found that changes in light scattering could be accompanied by changes in mitochondrial ultrastructure $^{13}$ without necessarily resulting in changes in morphology visible in fluorescence.

The spatial extent of the mitochondria-rich, abovethreshold $(>\mathrm{Th}=1.5)$ fluorescent segment is limited by optical resolution and imperfect thresholding and segmentation, and could include a small percentage of nonfluorescent or- ganelles surrounding the mitochondria. However, in our discussion so far, we have assumed that the potential presence of these organelles in the designated fluorescent regions would only add a static background based on the static scatter response of nonfluorescent organelles in the adjacent designated nonfluorescent regions $(\leqslant \mathrm{Th}=1.5)$. Still, it is possible that organelles that are structurally less active away from the mitochondria may be subject to mitochondrial signaling during apoptosis. Thus, when closely associated with mitochondria, some organelles (e.g., the endoplasmic reticulum) may change their structure during apoptosis, potentially giving rise to the OSIR changes observed in the designated fluorescent segments. This possibility may be unlikely, given the strong scattering contribution of mitochondria compared to other organelles. ${ }^{3}$ But to completely rule it out, the structural changes accounting for the OSIR decrease within the first hour of STS treatment remain to be fully elucidated. In conclusion, our results strongly point to the mitochondria as the likeliest source of the optical scatter changes measured in the first three hours of apoptosis induced by staurosporine. Mitochondria may give rise to these optical scatter changes directly through alterations in mitochondrial structure, or indirectly via signaling from mitochondria to organelles in their immediate vicinity.

\section{Acknowledgments}

This work was supported by NSF grant DBI-0852857.

\section{References}

1. N. N. Danial and S. J. Korsmeyer, "Cell death: critical control points," Cell 116, 205-219 (2004).

2. D.-F. Suen, K. L. Norris, and R. J. Youle, "Mitochondrial dynamics and apoptosis," Genes Dev. 22, 1577-1590 (2008).

3. J. D. Wilson, W. J. Cottrell, and T. H. Foster, "Index-of-refractiondependent subcellular light scattering observed with organellespecific dyes," J. Biomed. Opt. 12(1), 014010 (2007).

4. N. N. Boustany, Y. C. Tsai, B. Pfister, W. M. Joiner, G. A. Oyler, and N. V. Thakor, "BCL-x(L)-dependent light scattering by apoptotic cells," Biophys. J. 87(6), 4163-4171 (2004).

5. J. D. Wilson, B. R. Giesselman, S. Mitra, and T. H. Foster, "Lysosome-damage-induced scattering changes coincide with release of cytochrome c," Opt. Lett. 32(17), 2517-2519 (2007).

6. K. J. Chalut, J. H. Ostrander, M. G. Giacomelli, and A. Wax, "Light scattering measurements of subcellular structure provide noninvasive early detection of chemotherapy-induced apoptosis," Cancer Res. 69(3), 1199-1204 (2009)

7. N. N. Boustany, R. Drezek, and N. V. Thakor, "Calcium-induced alterations in mitochondrial morphology quantified in situ with optical scatter imaging," Biophys. J. 83, 1691-1700 (2002).

8. J. Y. Zheng, R. M. Pasternack, and N. N. Boustany, "Optical scatter imaging with a digital micromirror device," Opt. Express 17(22), 20401-20414 (2009).

9. N. N. Boustany, S. C. Kuo, and N. V. Thakor, "Optical scatter imaging: subcellular morphometry in situ with Fourier filtering," Opt. Lett. 26(14), 1063-1065 (2001).

10. D. Dunn, W. E. Higgins, and J. Wakeley, "Texture segmentation using 2-D Gabor elementary functions," IEEE Trans. Pattern Anal. Mach. Intell. 16(2), 130-149 (1994).

11. A. V. Loud, "A quantitative stereological description of the ultrastructure of normal liver parenchymal cells," J. Cell Biol. 37, 27-46 (1968).

12. R. M. Pasternack, J. Zheng, and N. N. Boustany, "Quantifying mitochondrial dynamics in apoptotic cells with optical Gabor-like filtering," in Biomedical Optics, OSA Technical Digest (CD), paper BTuE3, Optical Society of America, Washington, DC (2010).

13. J. Zheng, Y. Tsai, P. Kadimcherla, R. Zhang, J. Shi, G. A. Oyler, and N. N. Boustany, "The C-terminal transmembrane domain of Bcl-xL mediates changes in mitochondrial morphology," Biophys. J. 94(1), 286-297 (2008). 